\title{
EQUIDIVISIBLE PSEUDOVARIETIES OF SEMIGROUPS
}

\author{
JORGE ALMEIDA AND ALFREDO COSTA
}

\begin{abstract}
We give a complete characterization of pseudovarieties of semigroups whose finitely generated relatively free profinite semigroups are equidivisible. Besides the pseudovarieties of completely simple semigroups, they are precisely the pseudovarieties that are closed under Mal'cev product on the left by the pseudovariety of locally trivial semigroups. A further characterization which turns out to be instrumental is as the non-completely simple pseudovarieties that are closed under two-sided Karnofsky-Rhodes expansion.
\end{abstract}

\section{INTRODUCTION}

A pseudovariety of semigroups is a class of finite semigroups closed under taking subsemigroups, homomorphic images and finitary products. In the past few decades, pseudovarieties provided the main framework for the research on finite semigroups, motivated by Eilenberg's correspondence theorem between pseudovarieties and varieties of languages. In this context, the finitely generated relatively free profinite semigroups associated to each pseudovariety proved to be of fundamental importance. We assume the reader has some familiarity with this background. The books [19, 1, are indicated as supporting references. The paper [2] might also be useful for someone looking for a brief introduction.

In this paper we are concerned with equidivisible relatively free profinite semigroups. A semigroup $S$ is equidivisible if for every $u, v, x, y \in S$, the equality $u v=x y$ implies that $u=x$ and $v=y$, or that there is $t \in S$ such that $u t=x$ and $v=t y$, or such that $x t=u$ and $y=t v$. Equidivisible semigroups were introduced in 12 as a generalization of free semigroups. They were further investigated in 13 where a characterization of the completely simple semigroups as being a special class of equidivisible semigroups was given (cf. Theorem 5.1).

A pseudovariety of semigroups $\mathrm{V}$ is said to be equidivisible if every finitely generated free pro- $V$ semigroup is equidivisible. The paper [4] includes results about a special class of semigroup pseudovarieties, the WGGM pseudovarieties, the ones whose corresponding relatively free profinite semigroups are "weakly generalized group mapping". In the same paper, the WGGM condition is applied to obtain sufficient conditions for a pseudovariety to be finitely join irreducible in the lattice of ordered pseudovarieties. It is also

2010 Mathematics Subject Classification. Primary 20M07, $20 \mathrm{M} 05$.

Key words and phrases. semigroup, equidivisible, pseudovariety, Karnofsky-Rhodes expansion, connected expansion, two-sided Cayley graph.

Work partially supported respectively by CMUP (UID/MAT/00144/2013) and CMUC (UID/MAT/00324/2013), which are funded by FCT (Portugal) with national (MEC) and European structural funds (FEDER) under the partnership agreement PT2020. 
shown in [4] that a sufficient condition for a semigroup pseudovariety $\mathrm{V}$ to be WGGM is to be equidivisible and to contain LSI (the pseudovariety of finite semigroups whose local monoids are semillatices). This adds motivation to investigate the natural question: which pseudovarieties are equidivisible, other than those of completely simple semigroups?

In this paper we give a complete characterization of the equidivisible pseudovarieties of semigroups (Sec. 8), showing that those which are not contained in the pseudovariety of completely simple semigroups are precisely the ones of the form $\mathrm{V}=\mathrm{LI}(m \mathrm{~V}$, where $\mathrm{LI}$ denotes the pseudovariety of locally trivial semigroups and $(m$ denotes the Mal'cev product of pseudovarieties.

The semigroup pseudovarieties of the form $\mathrm{V}=\mathrm{A}(\mathrm{m} \mathrm{V}$, where $\mathrm{A}$ denotes the pseudovariety of aperiodic semigroups, are those whose corresponding variety of languages is closed under language concatenation [23, 6]. In [3, Lemma 4.8] it is shown that every such pseudovariety is equidivisible, with a proof that uses the closure under concatenation. In contrast to the approach made in [3] for this class of pseudovarieties, our proof of the equidivisibility of the pseudovarieties of the form $\mathrm{V}=\mathrm{LI}(\mathrm{m} \mathrm{V}$ does not use a characterization in terms of the corresponding varieties of languages, which are those that are closed under unambiguous product of languages [14, 15]. For our complete characterization of the equidivisible pseudovarieties, and in both directions of the proof, we had to use a distinct approach, based on the two-sided Karnofsky-Rhodes expansion of semigroups. This approach was inspired by the proof given in [18 that if a pseudovariety of semigroups $V$ is stable under (one-sided) right Karnofsky-Rhodes expansion, then the finitely generated free pro- $V$ semigroups have unambiguous $\leq_{\mathcal{R}}$-order. It turns out that, by a deep result of Rhodes et al. [20, 21], the pseudovarieties of the form $\mathrm{V}=\mathrm{LI} m \mathrm{~V}$ are precisely those that are stable under two-sided Karnofsky-Rhodes expansion (Corollary [3.3).

Roughly speaking, the two-sided Karnofsky-Rhodes expansion keeps track of the transition edges used to read a word in the two-sided Cayley graph of the semigroup expanded. If we only keep track of the strongly connected components, we obtain another expansion, which we call the two-sided connected expansion. We deduce from our main result that a pseudovariety is closed under two-sided Karnofsky-Rhodes expansion if and only if it is closed under two-sided connected expansion (cf. Corollary 8.4).

Another by-product of our results concerns the pseudovariety LG of finite semigroups whose local monoids are groups. After showing directly that the equidivisible subpseudovarieties of LG are precisely those containing LI, we apply our main result to deduce that, whenever $\mathrm{V}$ is a subpseudovariety of LG, the join LI $\vee \vee$ is equal to the Mal'cev product LI $m \vee$ (Corollary 8.6).

\section{Preliminaries}

Recall that [19, 1, 2] are our supporting references. We use the standard notation $\mathrm{V} \vee \mathrm{W}, \mathrm{V} * \mathrm{~W}, \mathrm{~V} * * \mathrm{~W}$ and $\mathrm{V} m \mathrm{~W}$ respectively for the join, the semidirect product, the two-sided semidirect product, and the Mal'cev product of pseudovarieties of semigroups. Occasionally (only in the preparatory 
Section 3) we refer to pseudovarieties of semigroupoids (namely the pseudovariety $\ell$ of trivial semigroupoids) and to varietal operations concerning them. We refer to [19, 2] for details.

The following pseudovarieties of semigroups appear in this paper:

- S: finite semigroups;

- A: finite aperiodic semigroups;

- G: finite groups;

- I: trivial semigroups;

- D: finite semigroups all of whose idempotents are right zeros;

- K: finite semigroups all of whose idempotents are left zeros;

- $\mathrm{LI}=\mathrm{K} \vee \mathrm{D}$ : finite semigroups whose local monoids are trivial;

- LG: finite semigroups whose local monoids are groups;

- LSI: finite semigroups whose local monoids are semilattices;

- CS: finite completely simple semigroups;

- CR: finite completely regular semigroups.

For a semigroup $S$, let $S^{I}$ be the monoid obtained from $S$ by adding an extra element $I$ which is the identity of $S^{I}$. This allows a convenient way of writing the definition of equidivisibility: the semigroup $S$ is equidivisible when, for every $u, v, x, y \in S$, the equality $u v=x y$ implies that there is $t \in S^{I}$ such that $u t=x$ and $v=t y$, or such that $x t=u$ and $y=t v$.

If $f: S \rightarrow T$ is a semigroup homomorphism, then we extend $f$ to a homomorphism from $S^{I}$ to $T^{I}$, also denoted $f$, by letting $f(I)=I$. Note that, for every alphabet $A$, the monoid $\left(A^{+}\right)^{I}$ can be identified with the free monoid $A^{*}$ in a natural manner. In particular, if $\varphi$ is a homomorphism from $A^{+}$to a semigroup $S$, then we have a unique extension of $\varphi$ to a homomorphism from $A^{*}$ to $S^{I}$, with $\varphi(1)=I$.

We were inspired by [18] in the use of semigroup expansions to obtain our main result. With this reference in mind (see also [10]), we quickly recall that, for a fixed alphabet $A$, the category of $A$-generated semigroups is the category $\mathcal{S}_{A}$ whose objects are the pairs of the form $(S, \varphi)$ in which $\varphi$ is an onto homomorphism $A^{+} \rightarrow S$, and where morphisms $(S, \varphi) \rightarrow(T, \psi)$ are the homomorphisms $\theta: S \rightarrow T$ such that $\theta \circ \varphi=\psi$. An expansion cut to generators defined in $\mathcal{S}_{A}$ is an endofunctor $F: \mathcal{S}_{A} \rightarrow \mathcal{S}_{A}$ equipped with a natural transformation from $F$ to the identity functor of $\mathcal{S}_{A}$. A convenient way to refer to $F$ is the notation correspondence $(S, \varphi) \mapsto\left(S^{F}, \varphi^{F}\right)$, where the pair $\left(S^{F}, \varphi^{F}\right)$ is the object $F(S, \varphi)$.

\section{The TWO-SIDED KARNOFSKY-RHODEs EXPANSION}

Let $\varphi$ be a homomorphism from $A^{+}$onto a semigroup $S$. The twosided Cayley graph defined by $\varphi$ is the directed graph $\Gamma_{\varphi}$ whose set of vertices is $S^{I} \times S^{I}$, and where an edge from $\left(s_{1}, t_{1}\right)$ to $\left(s_{2}, t_{2}\right)$ is a triple $\left(\left(s_{1}, t_{1}\right), a,\left(s_{2}, t_{2}\right)\right)$, with $a \in A$, such that $s_{1} \varphi(a)=s_{2}$ and $t_{1}=\varphi(a) t_{2}$. Giving to each edge $\left(\left(s_{1}, t_{1}\right), a,\left(s_{2}, t_{2}\right)\right)$ the label $a$, the graph $\Gamma_{\varphi}$ becomes a semi-automaton over the alphabet $A$. A labeling of paths is inherited from the labeling of edges in an obvious way. If $u \in A^{+}$, then we denote by $p_{\varphi, u}$, or simply $p_{u}$ if $\varphi$ is understood, the unique path from $(I, \varphi(u))$ to $(\varphi(u), I)$ labeled by $u$. 
For an edge $t$ in a directed graph $H$, we denote by $\alpha(t)$ its source and by $\omega(t)$ is target. The edge $t$ is a transition edge of $H$ if $\alpha(t)$ and $\omega(t)$ are not in the same strongly connected component of $H$. Returning our attention to the two-sided Cayley graph $\Gamma_{\varphi}$, for a path $p$ in $\Gamma_{\varphi}$, denote by $T(p)$ the set of transition edges in $p$. Let $\equiv_{\varphi}$ be the binary relation on $A^{+}$defined by $u \equiv_{\varphi} v$ if and only if $\varphi(u)=\varphi(v)$ and $T\left(p_{u}\right)=T\left(p_{v}\right)$. The relation $u \equiv_{\varphi} v$ is a congruence, a well-known fact whose routine proof is similar to the explicit proof we give later of an analogous result, Lemma 4.1. Denote by $S_{\varphi}^{K(\ell l)}$ the quotient $A^{+} / \equiv_{\varphi}$ and by $\varphi^{K(\ell l)}$ the corresponding quotient homomorphism $A^{+} \rightarrow S_{\varphi}^{K(\ell l)}$. For the sake of simplicity, we will write $S^{K(\ell l)}$ instead of $S_{\varphi}^{K(\ell l)}$, if the dependency on $\varphi$ is implicitly understood.

It is well known that the correspondence $(S, \varphi) \mapsto\left(S^{K(\ell l)}, \varphi^{K(\ell l)}\right)$ is an expansion cut to generators, which is called the two-sided Karnofsky-Rhodes expansion. There is an alternative way of defining this expansion, which puts it as a special case within a more general framework, and which we refer briefly, leaving the details for the bibliographic references supporting our discussion. The two-sided Karnofsky-Rhodes expansion is an example of a two-sided semidirect product expansion defined by a variety of semigroups (in this case, the variety of trivial semigroups), as introduced by Elston in [10]. In [18, a variation of this approach is followed, one where pseudovarieties of semigroupoids are used instead of varieties of semigroups. The notation $S^{K(\ell l)}$ is consistent with the notation used in [18] for the twosided semidirect product expansion $S^{K(\mathrm{~V})}$ of a profinite semigroup $S$ defined by a pseudovariety $\mathrm{V}$ of semigroupoids. As observed in [18, Sec. 10], if $\mathrm{V}$ is a pseudovariety of semigroups, then $S^{K(\ell \mathrm{V})}$ is the corresponding expansion $S^{K(\mathrm{~V})}$ introduced by Elston.

Suppose that the alphabet $A$ is finite. If $S$ is finite then $S^{K(\ell l)}$ is finite, because a kernel class of $\varphi^{K(\ell l)}$ is determined by a kernel class of $\varphi$ together with a set of transition edges of $\Gamma_{\varphi}$, and there is only a finite number of such classes and edges.

More generally, as explained in [18, Sec. 10], if $S$ is finite and $\mathrm{V}$ is a locally finite pseudovariety of semigroupoids (which is the case of $\ell \mathrm{l}$ ), then $S^{K(\mathrm{~V})}$ is a finite semigroup which is in a natural way a two-sided semidirect product $\left(\bar{\Omega}_{\Gamma_{\varphi}} \mathrm{V}\right) * * S$, and so if $S$ belongs to a pseudovariety $\mathrm{W}$ of semigroups, then $S^{K(\mathrm{~V})}$ belongs to $\mathrm{V} * * \mathrm{~W}$.

As remarked at the beginning of Section 11 of [18, the isomorphism between the quotient $A^{+} / \equiv_{\varphi}$ and the two-sided semidirect product $\left(\bar{\Omega}_{\Gamma_{\varphi}} \ell \mathrm{l}\right) * * S$ is justified by Tilson's result asserting that two paths in a graph $X$ coincide in the locally trivial free category generated by $X$ if and only if they have the same transition edges.

The following result is a special case of [19, Theorem 3.6.4].

Proposition 3.1. Let $\mathrm{W}$ be a locally finite pseudovariety and let $A$ be a finite alphabet. Then $\left(\bar{\Omega}_{A} \mathrm{~W}\right)^{K(\ell \mathrm{l})}$ is isomorphic to $\bar{\Omega}_{A}(\ell \mathrm{l} * * \mathrm{~W})$.

It is an easy exercise to show, directly from the definition we gave of the two-sided Karnofsky-Rhodes expansion, that if $\pi$ is the canonical projection $S^{K(\ell l)} \rightarrow S$ then the semigroup $\pi^{-1}(e)$ satisfies the identity $x y z=x z$, for every idempotent $e$ of $S$. Actually, as remarked in the proof of Lemma 3.4 
in [16], we have $\ell \mid * * \mathrm{~V} \subseteq \llbracket x y z=x z \rrbracket$ m $\mathrm{V}$, and so $\ell \mid * * \mathrm{~V} \subseteq \mathrm{LI}(m \mathrm{~V}$. Denote by $\bigcup_{n \geq 1} \ell \mid * *^{n} \vee$ the sequence of semigroup pseudovarieties recursively defined by

$$
\ell \mathbf{l} * *^{0} \mathrm{~V}=\mathrm{V}, \quad \ell \mathbf{l} * *^{n} \mathrm{~V}=\ell \mathbf{l} * *\left(\ell \mathbf{l} * *^{n-1} \mathrm{~V}\right), \quad n \geq 1
$$

The following theorem is a deep result of Rhodes et al. [20, 21] which the reader can find in [19, Corollary 5.3.22].

Theorem 3.2. Let $\mathrm{V}$ be a pseudovariety of semigroups. Then we have $\mathrm{LI}\left(\mathrm{m} \mathrm{V}=\bigcup_{n \geq 0} \ell \mathbf{l} * *^{n} \mathrm{~V}\right.$.

Say that a pseudovariety of semigroups $\mathrm{V}$ is closed under the two-sided Karnofsky-Rhodes expansion if we have $S_{\varphi}^{K(\ell \mathrm{l})} \in \mathrm{V}$ whenever $S \in \mathrm{V}$ and $\varphi$ is a homomorphism from a finitely generated free semigroup onto $S$. For the reader's convenience, we give a proof of the following easy consequence of Theorem 3.2 .

Corollary 3.3. A pseudovariety of semigroups $\mathrm{V}$ is closed under the twosided Karnofsky-Rhodes expansion if and only if $\mathrm{V}=\mathrm{LI}(\mathrm{m} \mathrm{V}$.

Proof. Suppose that $\mathrm{V}=\mathrm{LI}(\mathrm{m} \mathrm{V}$. Let $S \in \mathrm{V}$ and consider a homomorphism $\varphi: A^{+} \rightarrow S$. Using the aforementioned fact that $S_{\varphi}^{K(\ell l)} \in \llbracket x y z=x z \rrbracket @ \mathrm{~V}$, we immediately get that $S_{\varphi}^{K(\ell l)} \in \mathrm{V}$, because $\llbracket x y z=x z \rrbracket \subseteq$ LI. Alternatively, one can apply (the easy part of) Theorem 3.2, since $S_{\varphi}^{K(\ell)} \in \ell$ l ** V.

Conversely, suppose that $\mathrm{V}$ is closed under the two-sided KarnofskyRhodes expansion. Let $\mathrm{W}$ be a locally finite subpseudovariety of $\mathrm{V}$. Then $\bar{\Omega}_{A} \mathrm{~W}$ belongs to $\mathrm{V}$. By hypothesis, $\left(\bar{\Omega}_{A} \mathrm{~W}\right)^{K(\ell)}$ also belongs to $\mathrm{V}$. Applying Proposition [3.1, we conclude that $\ell \mathrm{l} * * \mathrm{~W} \subseteq \mathrm{V}$. As $\mathrm{W}$ can be any locally finite subpseudovariety of $\mathrm{V}$, we actually have $\ell+* \mathrm{~V} \subseteq \mathrm{V}$. We deduce from Theorem 3.2 that $\mathrm{LI}: \mathrm{V}=\mathrm{V}$.

\section{The TWO-SIDED CONNECTED EXPANSION}

In this section we show that the pseudovarieties closed under the two-sided Karnofsky-Rhodes expansion are equidivisible. Actually, it is not necessary to use the full force of the definition of the expansion. It suffices to use a weaker expansion which we introduce in this section.

Let $\varphi$ be a homomorphism from $A^{+}$onto a semigroup $S$. Given a path $p$ in the two-sided Cayley graph $\Gamma_{\varphi}$, denote by $C(p)$ the set of strongly connected components of $\Gamma_{\varphi}$ that contain some vertex in $p$. Let $\approx_{\varphi}$ be the binary relation on $A^{+}$defined by $u \approx_{\varphi} v$ if and only if $\varphi(u)=\varphi(v)$ and $C\left(p_{u}\right)=C\left(p_{v}\right)$.

Lemma 4.1. The relation $\approx_{\varphi}$ is a congruence.

Proof. The relation $\approx_{\varphi}$ is clearly an equivalence. Taking into account the symmetry of its definition, to prove that $\approx_{\varphi}$ is a congruence, it suffices to show that $C\left(p_{u w}\right)=C\left(p_{v w}\right)$ whenever $u, v, w \in A^{+}$are such that $u \approx_{\varphi} v$. Let $x$ be a vertex of $p_{u w}$ that is not in $p_{v w}$, where $u \approx_{\varphi} v$. Then, as $\varphi(u)=\varphi(v)$, we necessarily have $x=\left(\varphi\left(u_{1}\right), \varphi\left(u_{2} w\right)\right)$ for some $u_{1}, u_{2} \in A^{+}$ such that $u=u_{1} u_{2}$. Since $x^{\prime}=\left(\varphi\left(u_{1}\right), \varphi\left(u_{2}\right)\right)$ is a vertex of $p_{u}$, there is some vertex $x^{\prime \prime}$ in $p_{v}$ such that $x^{\prime}$ and $x^{\prime \prime}$ are in the same strongly connected component. Let $t$ be the label of a path from $x^{\prime}$ to $x^{\prime \prime}$, and let $z$ be the label 
of a path from $x^{\prime \prime}$ to $x^{\prime}$. We have $x^{\prime \prime}=\left(\varphi\left(v_{1}\right), \varphi\left(v_{2}\right)\right)$ for some $v_{1}, v_{2} \in A^{*}$ such that $v=v_{1} v_{2}$. Note that

$$
\varphi\left(u_{1} t\right)=\varphi\left(v_{1}\right), \varphi\left(u_{2}\right)=\varphi\left(t v_{2}\right), \varphi\left(v_{1} z\right)=\varphi\left(u_{1}\right), \varphi\left(v_{2}\right)=\varphi\left(z u_{2}\right) .
$$

Consider the vertex $x^{\prime \prime \prime}=\left(\varphi\left(v_{1}\right), \varphi\left(v_{2} w\right)\right)$. Looking at (4.1), we see that we also have $\varphi\left(u_{2} w\right)=\varphi\left(t v_{2} w\right)$ and $\varphi\left(v_{2} w\right)=\varphi\left(z u_{2} w\right)$, whence there is a path from $x$ to $x^{\prime \prime \prime}$ labeled $t$, and a path from $x^{\prime \prime \prime}$ to $x$ labeled $z$. This shows that $x^{\prime \prime \prime}$ belongs to the strongly connected component of $x$. Since $x^{\prime \prime \prime}$ belongs to $p_{v w}$, this establishes the inclusion $C\left(p_{u w}\right) \subseteq C\left(p_{v w}\right)$. Dually, we have $C\left(p_{v w}\right) \subseteq C\left(p_{u w}\right)$.

If $S$ is finite then $\approx_{\varphi}$ has finite index, because a $\approx_{\varphi}$-class is defined by a kernel class of $\varphi$ together with a set of strongly connected components of $\Gamma_{\varphi}$, and there is only a finite number of such classes and components.

We denote by $S^{C}$ the quotient $A^{+} / \approx_{\varphi}$ and by $\varphi^{C}$ the canonical homomorphism $A^{+} \rightarrow S^{C}$. Clearly, if $\varphi^{K(\ell l)}(u)=\varphi^{K(\ell l)}(v)$ then $\varphi^{C}(u)=\varphi^{C}(v)$, and so $S^{C}$ is a quotient of $S^{K(\ell l)}$. The semigroups $S^{C}$ and $S^{K(\ell l)}$ may not be isomorphic. For example, consider the onto homomorphism $\varphi$ from the two-letter alphabet $A=\{a, b\}$ onto the trivial semigroup $S$. Then $\varphi^{C}(a)=\varphi^{C}(b)$ but $\varphi^{K(\ell l)}(a) \neq \varphi^{K(\ell l)}(b)$.

Proposition 4.2. The correspondence $(S, \varphi) \mapsto\left(S^{C}, \varphi^{C}\right)$ is an expansion cut to generators.

Proof. Let $\varphi: A^{+} \rightarrow S$ and $\psi: A^{+} \rightarrow T$ be onto homomorphisms, and let $f: S \rightarrow T$ be a homomorphism such that $f \circ \varphi=\psi$. The mapping $f$ induces a homomorphism $\bar{f}$ of semi-automata from $\Gamma_{\varphi}$ to $\Gamma_{\psi}$, defined by the following mappings from vertices and edges of $\Gamma_{\varphi}$ respectively to vertices and edges of $\Gamma_{\psi}$ :

$$
(s, t) \mapsto(f(s), f(t)), \quad\left((s, t), a,\left(s^{\prime}, t^{\prime}\right)\right) \mapsto\left((f(s), f(t)), a,\left(f\left(s^{\prime}\right), f\left(t^{\prime}\right)\right)\right) .
$$

Let $u, v \in A^{+}$be such that $u \approx_{\varphi} v$. Then we have $\varphi(u)=\varphi(v)$ and $\psi(u)=\psi(v)$. Let $y=\left(\psi\left(u_{1}\right), \psi\left(u_{2}\right)\right)$ be a vertex in $p_{u}^{\psi}$, where $u=u_{1} u_{2}$, with $u_{1}, u_{2} \in A^{*}$. Then $y=\bar{f}(x)$, where $x=\left(\varphi\left(u_{1}\right), \varphi\left(u_{2}\right)\right)$ is a vertex in $p_{u}^{\varphi}$. Since $u \approx_{\varphi} v$, there is a vertex $x^{\prime}$ in $p_{v}^{\varphi}$ such that $x$ and $x^{\prime}$ are in the same strongly connected component of $\Gamma_{\varphi}$. Clearly, $y=\bar{f}(x)$ and $y^{\prime}=\bar{f}\left(x^{\prime}\right)$ are in the same strongly connected component of $\Gamma_{\psi}$. As $x^{\prime}$ is in $p_{v}^{\varphi}$, we have $y^{\prime}$ in $p_{v}^{\psi}$, showing that $C\left(p_{u}^{\psi}\right) \subseteq C\left(p_{v}^{\psi}\right)$. By symmetry, we have $C\left(p_{u}^{\psi}\right) \supseteq C\left(p_{v}^{\psi}\right)$. This establishes the equality $u \approx_{\psi} v$, and therefore we may consider the unique semigroup homomorphism $f^{C}: S^{C} \rightarrow T^{C}$ such that $f^{C} \circ \varphi^{C}=\psi^{C}$.

Let us call the expansion $(S, \varphi) \mapsto\left(S^{C}, \varphi^{C}\right)$ the two-sided connected expansion.

A pseudovariety of semigroups $\mathrm{V}$ is closed under two-sided connected expansion if we have $S^{C}$ whenever $S \in \mathrm{V}$.

Proposition 4.3. If $\vee$ is a pseudovariety of semigroups closed under twosided connected expansion then $\mathrm{V}$ is equidivisible. 
Proof. Let $A$ be a finite alphabet. Suppose $u, v, x, y$ are elements of $\bar{\Omega}_{A} \mathrm{~V}$ such that $u v=x y$. Let $\Phi$ be a continuous homomorphism onto a semigroup $S$ from $\mathrm{V}$, and let $\varphi$ be its restriction to $A^{+}$. Denote by $\Phi^{C}$ the unique continuous homomorphism from $\bar{\Omega}_{A} \vee$ onto $S^{C}$ whose restriction to $A^{+}$coincides with $\varphi^{C}$. Consider elements $u_{0}, v_{0}, x_{0}, y_{0}$ of $A^{+}$such that $\varphi^{C}\left(u_{0}\right)=$ $\Phi^{C}(u), \varphi^{C}\left(v_{0}\right)=\Phi^{C}(v), \varphi^{C}\left(x_{0}\right)=\Phi^{C}(x)$ and $\varphi^{C}\left(y_{0}\right)=\Phi^{C}(y)$. The vertex $(\Phi(u), \Phi(v))=\left(\varphi\left(u_{0}\right), \varphi\left(v_{0}\right)\right)$ belongs to the path $\left(I, \varphi\left(u_{0} v_{0}\right)\right) \stackrel{u_{0} v_{0}}{\longrightarrow}$ $\left(\varphi\left(u_{0} v_{0}\right), I\right)$ of the two-sided Cayley graph $\Gamma_{\varphi}$. Since $u v=x y$, we know that $\Phi^{C}(u v)=\Phi^{C}(x y)$. Therefore, there is a vertex $(r, s)$ in the path $\left(I, \varphi\left(u_{0} v_{0}\right)\right) \stackrel{x_{0} y_{0}}{\longrightarrow}\left(\varphi\left(u_{0} v_{0}\right), I\right)$ of $\Gamma_{\varphi}$ which lies in the strongly connected component of $(\Phi(u), \Phi(v))$. Since $(\Phi(x), \Phi(y))$ is clearly also in the path $\left(I, \varphi\left(u_{0} v_{0}\right)\right) \stackrel{x_{0} y_{0}}{\longrightarrow}\left(\varphi\left(u_{0} v_{0}\right), I\right)$, we conclude that in $\Gamma_{\varphi}$ there is a (possibly empty) path from $(\Phi(u), \Phi(v))$ to $(\Phi(x), \Phi(y))$ or a path from $(\Phi(x), \Phi(y))$ to $(\Phi(u), \Phi(v))$.

Therefore, there is a word $t_{\Phi} \in A^{*}$ such that

$$
\left\{\begin{array}{l}
\Phi\left(u t_{\Phi}\right)=\Phi(x) \\
\Phi(v)=\Phi\left(t_{\Phi} y\right),
\end{array}\right.
$$

in which case we say that $\Phi$ is of the first type, or there is a word $\tau_{\Phi} \in A^{*}$ such that

$$
\left\{\begin{array}{l}
\Phi\left(x \tau_{\Phi}\right)=\Phi(u) \\
\Phi(y)=\Phi\left(\tau_{\Phi} v\right),
\end{array}\right.
$$

in which we say that $\Phi$ is of the second type. Note that $\Phi$ can be simultaneously of the first type and of the second type. The result now follows from a standard argument, which we write down for the reader's convenience. We know that $\bar{\Omega}_{A} \mathrm{~V}$ is the inverse limit of an inverse system of semigroups from $\mathrm{V}$ defined by a countable set of connecting onto homomorphisms of the form $\pi_{m, n}: S_{m} \rightarrow S_{n}$, where $m, n$ are arbitrary positive integers with $m \geq n$. For each $n \geq 1$, let $\pi_{n}$ be the projection $\bar{\Omega}_{A} \vee \rightarrow S_{n}$ associated to this inverse system. Note that, for each $n \leq m$, the homomorphism $\pi_{n}$ is of the same type as $\pi_{m}$. On the other hand, since there are only two types, at least one of them occurs infinitely often. Combining these two simple observations we conclude that $\pi_{n}$ is of the first type for every $n \geq 1$, or of the second type for every $n \geq 1$. Without loss of generality, we assume the former case. Denote $t_{\pi_{n}}$ by $t_{n}$. We have

$$
\pi_{n}\left(u t_{n}\right)=\pi_{n}(x) \text { and } \pi_{n}(v)=\pi_{n}\left(t_{n} y\right),
$$

for every $n \geq 1$. Let $t$ be an accumulation point in $\left(\bar{\Omega}_{A} \mathrm{~V}\right)^{I}$ of the sequence $\left(t_{n}\right)_{n}$. Fix $k \geq 1$, and let $n \geq k$. Applying $\pi_{n, k}$ to (4.2), we get

$$
\pi_{k}\left(u t_{n}\right)=\pi_{k}(x) \text { and } \pi_{k}(v)=\pi_{k}\left(t_{n} y\right),
$$

for every $n \geq k$. By continuity of $\pi_{k}$, we obtain

$$
\pi_{k}(u t)=\pi_{k}(x) \quad \text { and } \quad \pi_{k}(v)=\pi_{k}(t y) .
$$

This implies $u t=x$ and $v=t y$. The case where $\pi_{n}$ is of the second type for every $n \geq 1$ leads to the existence of $\tau$ in $\left(\bar{\Omega}_{A} \mathrm{~V}\right)^{I}$ such that $x \tau=u$ and $y=\tau v$. 
Corollary 4.4. If $\mathrm{V}$ is a pseudovariety of semigroups closed under twosided Karnofsky-Rhodes expansion then $\mathrm{V}$ is equidivisible.

Proof. It follows immediately from Proposition 4.3 and the fact that the two-sided connected expansion of a semigroup $S$ is a homomorphic image of the two-sided Karnofsky-Rhodes expansion of $S$.

Corollary 4.5. If $\mathrm{V}$ is a pseudovariety of semigroups such that $\mathrm{V}=\mathrm{LI} m \mathrm{~V}$ then $\mathrm{V}$ is equidivisible.

Proof. Apply Corollary 4.4 and (the easy part of) Corollary 3.3 .

\section{Equidivisible SubPSEUdovarieties OF CR}

It was proved in 13 that every completely simple semigroup is equidivisible. In fact, the following stronger result was established.

Theorem 5.1. A semigroup $S$ is completely simple if and only if for every $u, v, x, y \in S$, the equality $u v=x y$ implies the existence of $t_{1}, t_{2} \in S^{I}$ such that $u t_{1}=x, t_{1} y=v, u=x t_{2}$, and $y=t_{2} v$.

On the other hand we have the following simple fact.

Lemma 5.2. If $\vee$ is a pseudovariety of completely regular semigroups containing $\mathrm{SI}$ then $\mathrm{V}$ is not equidivisible.

Proof. Consider the alphabet $A=\{a, b\}$. We claim that $\bar{\Omega}_{A} \vee$ is not equidivisible. Indeed, we have $a b \cdot(a b)^{\omega}=a \cdot b$. On the other hand, since $c(a b) \nsubseteq c(a)$, there is no $t \in\left(\bar{\Omega}_{A} \mathrm{~V}\right)^{I}$ such that $a b \cdot t=a$. Similarly, there is no $t \in\left(\bar{\Omega}_{A} \mathrm{~V}\right)^{I}$ such that $t \cdot(a b)^{\omega}=b$. This establishes the claim.

Since a pseudovariety of completely regular semigroups not containing SI is contained in CS, combining Theorem 5.1 and Lemma 5.2 we deduce the following result.

Corollary 5.3. A pseudovariety of completely regular semigroups is equidivisible if and only if it is contained in CS.

\section{LETTER SUPER-CANCELABILITY AS A NECESSARY CONDITION FOR EQUIDIVISIBILITY}

Let $S$ be an $A$-generated compact semigroup. This implies $S=S^{I} A=$ $A S^{I}$. Say that $S$ is right letter cancelative when, for every $a \in A$ and $u, v \in S^{I}$, the equality $u a=v a$ implies $u=v$. Say moreover that $S$ is right letter super-cancelative when, for every $a, b \in A$ and $u, v \in S^{I}$, the equality $u a=v b$ implies $a=b$ and $u=v$. We have the obvious dual notions of left letter cancelative and left letter super-cancelative semigroup. If $S$ is simultaneously right and left letter (super-)cancelative, then we say $S$ is letter (super-)cancelative.

Say that a pseudovariety of semigroups $\mathrm{V}$ is right letter (super-)cancelative if $\bar{\Omega}_{A} \mathrm{~V}$ is right letter (super-)cancelative, for every finite alphabet $A$. One also has the dual notions of left letter (super-)cancelative and letter (super-) cancelative pseudovariety. 
Example 6.1. If $\mathrm{V}$ is a semigroup pseudovariety containing some nontrivial monoid and such that $\mathrm{V}=\mathrm{V} * \mathrm{D}$, then $\mathrm{V}$ is letter super-cancelative (cf. 7, Prop. 1.60] and [1, Exercise 10.2.10]).

In [4] one finds a characterization of the (right/left) super-cancelative pseudovarieties as a routine exercise of application of basic results in the theory of profinite semigroups. The following simple observation is included in that characterization.

Lemma 6.2. A semigroup pseudovariety $\mathrm{V}$ is right letter super-cancelative if and only if $\mathrm{D} \subseteq \mathrm{V}$ and $\mathrm{V}$ is right letter cancelative. Dually, $\mathrm{V}$ is left letter super-cancelative if and only if $\mathrm{K} \subseteq \mathrm{V}$ and $\mathrm{V}$ is left letter cancelative. Therefore, $\mathrm{V}$ is letter super-cancelative if and only if $\mathrm{LI} \subseteq \mathrm{V}$ and $\mathrm{V}$ is letter cancelative.

Letter super-cancelability appears as a necessary condition for equidivisibility in the following way.

Proposition 6.3. If $\mathrm{V}$ is an equidivisible pseudovariety of semigroups not contained in $\mathrm{CS}$ then $\mathrm{V}$ is letter supper-cancelative.

Proof. Fix a finite alphabet $A$. Let $u, v \in\left(\bar{\Omega}_{A} S\right)^{I}$ and let $a, b \in A$ be such that $\mathrm{V} \models u a=v b$. Since $\bar{\Omega}_{A} \mathrm{~V}$ is equidivisible, there is $t \in\left(\bar{\Omega}_{A} \mathrm{\vee}\right)^{I}$ such that $\mathrm{V} \models u t=v$ and $\mathrm{V} \models a=t b$, or such that $\mathrm{V} \models v t=u$ and $\mathrm{V} \models b=t a$. Suppose that $t \neq I$. Suppose also that $\mathrm{V} \models a=t b$. Replacing by $a$ every letter occurring in $t b$, we get $\mathrm{V} \models a=a^{\nu}$ for some profinite exponent $\nu>1$. This implies that $\mathrm{V} \subseteq \mathrm{CR}$. Corollary 5.3 states that the equidivisible subpseudovarieties of CR are precisely the subpseudovarieties of CS. Since $\mathrm{V}$ is not contained in CS, we reach a contradiction. Similarly, $\mathrm{V} \models a=t b$ leads to a contradiction. To avoid the contradiction, we must have $t=I$, whence $a=b$ and $\mathrm{V} \models u=v$. Symmetrically, $\mathrm{V} \models a u=b v$ implies $a=b$ and $\mathrm{V} \models u=v$.

Combining Proposition 6.3 with Lemma 6.2 we get the following corollary.

Corollary 6.4. If $\mathrm{V}$ is an equidivisible pseudovariety not contained in $\mathrm{CS}$, then $\mathrm{V}$ contains $\mathrm{LI}$.

\section{Equidivisible SUbPSEUdovarieties of LG}

Recall that the class LG of all finite local groups is the largest pseudovariety of semigroups whose semilattices are trivial. Local groups are thus generalizations of completely simple semigroups that turn out to be sometimes much harder to handle. For our purposes, the following technical result turns out to be essential.

Lemma 7.1. If $\mathrm{V}$ is a subpseudovariety of $\mathrm{LG}$ containing $\mathrm{D}$, then $\mathrm{V}$ is right letter super-cancelative.

Proof. Let $u$ and $v$ be pseudowords and $a$ and $b$ be letters such that the pseudoidentity $u a=v b$ holds in $\mathrm{V}$. The pseudovariety $\mathrm{D}$ is right letter super-cancelative, whence $a=b$ and $\mathrm{D}$ satisfies $u=v$. In particular, $u=v$ or both $u$ and $v$ are infinite pseudowords. Suppose the latter case occurs. Let $s_{n}$ be the suffix of $u$ of length $n$, which is also the suffix of length $n$ of $v$, 
that is, there are factorizations $u=u_{n} s_{n}$ and $v=v_{n} s_{n}$. By compactness, there exists a convergent subsequence of the sequence of triples $\left(u_{n}, v_{n}, s_{n}\right)$ and, therefore, there exist pseudowords $u^{\prime}, v^{\prime}, w$, where $w$ is infinite, such that $u=u^{\prime} w$ and $v=v^{\prime} w$ (in S). Since $w$ is infinite, there is a factorization of the form $w=w_{1} w_{2}^{\omega} w_{3}$ [1, Corollary 5.6.2]. As $\vee$ is contained in LG, it must satisfy the following pseudoidentities:

$$
w=w_{1} w_{2}^{\omega} w_{3}=w_{1}\left(w_{2}^{\omega} w_{3} a w_{2}^{\omega}\right)^{\omega} w_{3}=w_{1} w_{2}^{\omega} w_{3} a t=w a \cdot t
$$

where $t=\left(w_{2}^{\omega} w_{3} a w_{2}^{\omega}\right)^{\omega-1} w_{3}$. Hence, since the pseudovariety $\vee$ satisfies $u a=v a$, it also satisfies

$$
u=u^{\prime} w=u^{\prime} w a \cdot t=u a \cdot t=v a \cdot t=v^{\prime} w a \cdot t=v^{\prime} w=v .
$$

Theorem 7.2. A subpseudovariety of LG is equidivisible if and only if it is contained in $\mathrm{CS}$ or it contains $\mathrm{LI}$.

Proof. We already know that every subpseudovariety of CS is equidivisible by Theorem 5.1. Thus, for the remainder of the proof, we assume that $\mathrm{V}$ is a subpseudovariety of LG not contained in CS.

If $\mathrm{V}$ is equidivisible, then $\mathrm{V}$ contains $\mathrm{LI}$ by Corollary 6.4. For the converse, suppose that $\mathrm{V}$ contains $\mathrm{LI}$ and that $\mathrm{V}$ satisfies the pseudoidentity $u v=x y$. By Lemma 7.1, $\mathrm{V}$ is right letter super-cancelative. By duality, $\mathrm{V}$ is also left letter super-cancelative. Therefore, to prove that there is a (possibly empty) pseudoword $t$ such that in $\mathrm{V}$ we have $u t=x$ and $v=t y$, or $x t=u$ and $y=t v$, we may assume that all the pseudowords $u, v, x, y$ are infinite. Hence, $u v=x y$ may be viewed as an equality between products in the minimum ideal of a suitable $\bar{\Omega}_{A} \mathrm{~V}$, which is a completely simple semigroup. By Theorem 5.1, we may conclude that $\mathrm{V}$ is equidivisible.

\section{Characterization of EQuidivisible pseudovarieties}

Let $\varphi$ be a homomorphism from $A^{+}$onto a semigroup $S$. Given $u \in A^{+}$, a transition edge for $u$ in $\Gamma_{\varphi}$ is an element of $T\left(p_{u}\right)$. Note that $T\left(p_{u}\right)$ is always nonempty, since there is no path from $(\varphi(u), I)$ to $(I, \varphi(u))$ in $\Gamma_{\varphi}$. If $A$ and $S$ are finite, then $T\left(p_{u}\right)$ is finite and so, for some integer $n$, we can consider the sequence $\left(\varepsilon_{i}\right)_{i \in\{1, \ldots, n\}}$ of transition edges for $u$ in $\Gamma_{\varphi}$, where $\varepsilon_{i}$ is the $i$-th transition edge appearing in $p_{u}$.

In this section we shall work primarily with the expansion $\varphi^{K(\ell l)}$, but at some point it will be convenient to use another expansion which we next describe. In a graph, the content of a path $p$ is the set $c(p)$ of edges in the path. Consider the relation $\equiv_{\varphi, \mathrm{SI}}$ on $A^{+}$defined by $u \equiv_{\varphi, \mathrm{SI}} v$ if and only if $c\left(p_{u}\right)=c\left(q_{v}\right)$. Note that since $p_{w}$ starts at $(I, \varphi(w))$, if $u \equiv_{\varphi, \mathrm{SI}} v$ then $\varphi(u)=\varphi(v)$ holds. The relation $\equiv_{\varphi, \mathrm{SI}}$ is a congruence and the quotient homomorphism $A^{+} \rightarrow A^{+} / \equiv \varphi, \mathrm{Sl}$ is precisely the two-sided semidirect product expansion $\varphi^{K(\ell \mathrm{SI})}: A^{+} \rightarrow S^{K(\ell \mathrm{SI})}(\mathrm{cf}$. [10, Sec. 5.4])!

\footnotetext{
${ }^{1}$ The reader is cautioned for some misprints in the discussion made in [10, Sec. 5.4]; for instance, at some point a map $\psi$ is defined that takes a word $u$ to the set of edges in $p_{u}$, and not to the set of transition edges as by mistake it is written there. The characterization of $\varphi^{K(\ell S I)}$ is an application of [10, Corollary 5.4] and of a result of I. Simon [22] (a proof of which may be found in 9, Theorem VIII.7.1]) stating that two paths in a graph have the same content if and only if they are equal in the free category relatively to $\ell \mathrm{SI}$.
} 
Suppose moreover that $A$ and $S$ are finite. Then $S^{K(\ell l)}$ and $S^{K(\ell S I)}$ are both finite semigroups. Denote by $\Phi$ (respectively, $\Phi^{K(\ell l)}$ and $\Phi^{K(\ell S I)}$ ) the unique continuous homomorphism from $\bar{\Omega}_{A} \mathrm{~S}$ onto $S$ (respectively, $S^{K(\ell)}$ and $S^{K(\ell \mathrm{SI})}$ ) whose restriction to $A^{+}$is $\varphi$ (respectively, $\varphi^{K(\ell \mathrm{l})}$ and $\left.\varphi^{K(\ell \mathrm{SI})}\right)$. Let $u \in \bar{\Omega}_{A} \mathrm{~S}$. Consider an arbitrary sequence $\left(u_{n}\right)_{n}$ of elements of $A^{+}$converging to $u$. Then, there is $N$ such that $\Phi^{K(\ell l)}(u)=\varphi^{K(\ell l)}\left(u_{n}\right)$ for every $n \geq N$. Therefore, we can define a transition edge for $u$ in $\Gamma_{\varphi}$ as being an element of $T\left(p_{u_{n}}\right)$ for every sufficiently large $n$, since this set depends only on $\varphi$ and $u$. In a similar way, one can define the sequence of transition edges for $u$ in $\Gamma_{\varphi}$ as being the sequence of transition edges for $u_{n}$ in $\Gamma_{\varphi}$ for every sufficiently large $n$, and an edge for $u$ in $\Gamma_{\varphi}$ as being an element of $c\left(p_{u_{n}}\right)$ for every sufficiently large $n$. Note that a transition edge for $u$ is indeed an edge for $u$.

If $\psi$ is a continuous homomorphism from $\bar{\Omega}_{A}$ S onto a finite semigroup $T$, then we denote by $\Gamma_{\psi}$ the two-sided Cayley graph of the restriction of $\psi$ to $A^{+}$. Since $\psi$ is the unique continuous extension to $\bar{\Omega}_{A} \mathrm{~S}$ of its restriction to $A^{+}$, the homomorphisms $\psi^{K(\ell \mathrm{l})}$ and $\psi^{K(\ell \mathrm{SI})}$ are defined in view of the previous paragraph. Their images are also denoted $S_{\varphi}^{K(\ell \mathrm{I})}$ and $S_{\psi}^{K(\ell \mathrm{SI})}$, respectively.

Lemma 8.1. Let $\varphi$ be a continuous homomorphism from $\bar{\Omega}_{A} \mathrm{~S}$ onto a finite semigroup $S$, where $A$ is a finite alphabet. Let $u \in \bar{\Omega}_{A} \mathrm{~S}$. If $\left(\left(s_{1}, t_{1}\right), a,\left(s_{2}, t_{2}\right)\right)$ is an edge for $u$ in $\Gamma_{\varphi}$, then there is a factorization $u=u_{1} a u_{2}$ of $u$, with $u_{1}, u_{2} \in\left(\bar{\Omega}_{A} \mathrm{~S}\right)^{I}$, such that $\varphi\left(u_{1}\right)=s_{1}$ and $\varphi\left(u_{2}\right)=t_{2}$.

Proof. We may consider a sequence $\left(u_{n}\right)_{n}$ of elements of $A^{+}$converging to $u$ and such that $\varphi^{K(\ell S I)}\left(u_{n}\right)=\varphi^{K(\ell S I)}(u)$ for every $n$. In particular, for every $n$, the edge $\left(\left(s_{1}, t_{1}\right), a,\left(s_{2}, t_{2}\right)\right)$ is an edge for $u_{n}$ in $\Gamma_{\varphi}$, and so $u_{n}$ factors as $u_{n}=u_{n, 1} a u_{n, 2}$ for some $u_{n, 1}, u_{n, 2} \in A^{*}$ such that $\varphi\left(u_{n, 1}\right)=s_{1}$ and $\varphi\left(u_{n, 2}\right)=t_{2}$. By compactness, the sequence of pairs $\left(u_{n, 1}, u_{n, 2}\right)$ has some accumulation point $\left(u_{1}, u_{2}\right)$ in $\left(\bar{\Omega}_{A} S\right)^{I} \times\left(\bar{\Omega}_{A} S\right)^{I}$. By continuity of multiplication and of $\varphi$, we have $u=u_{1} a u_{2}, \varphi\left(u_{1}\right)=s_{1}$ and $\varphi\left(u_{2}\right)=t_{2}$.

Lemma 8.2. Let $\theta: B^{+} \rightarrow A^{+}$be a homomorphism satisfying $\theta(B)=A$, for some alphabets $A$ and $B$. Consider a homomorphism $\varphi$ from $A^{+}$onto a semigroup $S$. Let $\psi$ be the homomorphism from $B^{+}$onto $S$ such that $\psi=\varphi \circ \theta$. Then we have

$$
\psi^{K(\ell l)}(u)=\psi^{K(\ell l)}(v) \Longrightarrow \varphi^{K(\ell l)}(\theta(u))=\varphi^{K(\ell l)}(\theta(v)),
$$

for every $u, v \in B^{+}$. Consequently, $S_{\varphi}^{K(\ell \mathrm{l})}$ is a homomorphic image of $S_{\psi}^{K(\ell \mathrm{l})}$.

Proof. The proof relies on the following fact: there is in $\Gamma_{\psi}$ a path from $\left(s_{1}, t_{1}\right)$ to $\left(s_{2}, t_{2}\right)$ labeled by $u \in B^{+}$if and only if there is in $\Gamma_{\varphi}$ a path from $\left(s_{1}, t_{1}\right)$ to $\left(s_{2}, t_{2}\right)$ labeled by $\theta(u) \in A^{+}$. In particular, $\left(\left(s_{1}, t_{1}\right), b,\left(s_{2}, t_{2}\right)\right)$ is a transition edge of $\Gamma_{\psi}$ if and only if $\left(\left(s_{1}, t_{1}\right), \theta(b),\left(s_{2}, t_{2}\right)\right)$ is a transition edge of $\Gamma_{\varphi}$.

Let $u, v \in B^{+}$be such that $\psi^{K(\ell l)}(u)=\psi^{K(\ell l)}(v)$. Then we immediately have $\varphi(\theta(u))=\varphi(\theta(v))$. Suppose that $\left(\left(s_{1}, t_{1}\right), a,\left(s_{2}, t_{2}\right)\right)$ is a transition edge of $\Gamma_{\varphi}$ belonging to the path $p_{\theta(u)}$. We have $s_{1}=\varphi\left(w_{1}\right)$ and $t_{2}=\varphi\left(w_{2}\right)$ for some $w_{1}, w_{2} \in A^{*}$ such that $\theta(u)=w_{1} a w_{2}$. There is a factorization 
$u=u_{1} b u_{2}$ with $w_{1}=\theta\left(u_{1}\right), w_{2}=\theta\left(u_{2}\right)$ and $a=\theta(b)$. It then follows that $\left(\left(s_{1}, t_{1}\right), b,\left(s_{2}, t_{2}\right)\right)$ is a transition edge of $\Gamma_{\psi}$ belonging to $p_{u}$. It belongs also to $p_{v}$, since $\psi^{K(\ell l)}(u)=\psi^{K(\ell l)}(v)$. Therefore, $\left(\left(s_{1}, t_{1}\right), a,\left(s_{2}, t_{2}\right)\right)$ is a transition edge of $\Gamma_{\varphi}$ in $p_{\theta(v)}$. Symmetrically, every transition edge of $\Gamma_{\varphi}$ belonging to $p_{\theta(v)}$ also belongs to $p_{\theta(u)}$, establishing that $\varphi^{K(\ell l)}(\theta(u))=$ $\varphi^{K(\ell l)}(\theta(v))$.

Therefore, we can consider the onto homomorphism $\rho: S_{\psi}^{K(\ell \mathrm{l})} \rightarrow S_{\varphi}^{K(\ell \mathrm{l})}$ defined by $\rho\left(\psi^{K(\ell l)}(u)\right)=\varphi^{K(\ell l)}(\theta(u))$.

We are now ready to prove our main result.

Theorem 8.3. A pseudovariety of semigroups $\mathrm{V}$ is equidivisible if and only if it is contained in CS or it is closed under the two-sided Karnofsky-Rhodes expansion.

Proof. The "if" part follows from Corollaries 4.4 and 5.3 ,

Conversely, suppose that $\mathrm{V}$ is an equidivisible pseudovariety not contained in CS. Let $u, v$ be elements of $\bar{\Omega}_{X} \mathrm{~S}$ such that $\mathrm{V} \models u=v$, where $X$ is some finite alphabet. For a finite alphabet $A$, let $\varphi$ be a continuous homomorphism from $\bar{\Omega}_{A} S$ onto a semigroup $S$ from $\mathrm{V}$. Consider the finite alphabet $B=A \cup X$. There is a continuous onto homomorphism $\theta: \bar{\Omega}_{B} \mathrm{~S} \rightarrow \bar{\Omega}_{A} \mathrm{~S}$ such that $\theta(B)=A$. Let $\psi$ be the unique continuous homomorphism from $\bar{\Omega}_{B} \mathrm{~S}$ onto $S$ such that $\psi=\varphi \circ \theta$. We will show that $S_{\psi}^{K(\ell l)} \models u=v$.

Viewing $u, v$ as elements of $\bar{\Omega}_{B} S$, and because $\mathrm{V} \models u=v$, we have $\psi(u)=\psi(v)$. We claim that $\psi^{K(\ell \mathrm{l})}(u)=\psi^{K(\ell \mathrm{l})}(v)$. Let $\left(\varepsilon_{i}\right)_{i \in\{1, \ldots, n\}}$ and $\left(\delta_{i}\right)_{i \in\{1, \ldots, m\}}$ be the sequences of transition edges in $\Gamma_{\psi}$ respectively for $u$ and for $v$. Without loss of generality, we may assume that $n \leq m$.

Suppose that the set

$$
\left\{i \in\{1, \ldots, n\} \mid \varepsilon_{i} \neq \delta_{i}\right\}
$$

is nonempty, and let $j$ be its minimum. By Lemma 8.1 , there are factorizations $u=u_{1} a u_{2}$ of $u$ and $v=v_{1} b v_{2}$ of $v$, with $a, b \in B$ and $u_{1}, u_{2}, v_{1}, v_{2} \in$ $\left(\bar{\Omega}_{B} S\right)^{I}$, such that

$$
\varepsilon_{j}=\left(\left(\psi\left(u_{1}\right), \psi\left(a u_{2}\right)\right), a,\left(\psi\left(u_{1} a\right), \psi\left(u_{2}\right)\right)\right.
$$

and

$$
\delta_{j}=\left(\left(\psi\left(v_{1}\right), \psi\left(b v_{2}\right)\right), b,\left(\psi\left(v_{1} b\right), \psi\left(v_{2}\right)\right) .\right.
$$

Note that $\alpha\left(\varepsilon_{j}\right)$ and $\alpha\left(\delta_{j}\right)$ belong to the same strongly connected component of $\Gamma_{\psi}$, by the minimality of the index $j$.

Since $\mathbf{V} \models u_{1} a \cdot u_{2}=v_{1} \cdot b v_{2}$ and $\mathbf{V}$ is equidivisible, there is $t \in\left(\bar{\Omega}_{B} \mathrm{~S}\right)^{I}$ such that

$$
\mathrm{V} \models u_{1} a t=v_{1} \quad \text { and } \quad \mathrm{V} \models u_{2}=t b v_{2},
$$

or

$$
\mathrm{V} \models v_{1} t=u_{1} a \quad \text { and } \quad \mathrm{V} \models b v_{2}=t u_{2} .
$$

If Case (8.2) holds, then there is in $\Gamma_{\psi}$ a (possibly empty) path from $\omega\left(\varepsilon_{j}\right)$ to $\alpha\left(\delta_{j}\right)$, labeled by a word $t_{0} \in B^{*}$ such that $\psi\left(t_{0}\right)=\psi(t)$. Since $\alpha\left(\delta_{j}\right)$ and $\omega\left(\delta_{j}\right)$ belong to the same strongly connected component, we conclude 
there is in $\Gamma_{\psi}$ a path from $\omega\left(\varepsilon_{j}\right)$ to $\alpha\left(\varepsilon_{j}\right)$, contradicting the fact that $\varepsilon_{j}$ is a transition edge.

Therefore, Case (8.3) holds with $t \neq I$. By Proposition 6.3, it follows from (8.3) that there is $t^{\prime} \in\left(\bar{\Omega}_{B} S\right)^{I}$ with $t=t^{\prime} a$ and

$$
\mathrm{V} \models v_{1} t^{\prime}=u_{1} \quad \text { and } \quad \mathrm{V} \models b v_{2}=t^{\prime} a u_{2} .
$$

Suppose that $t^{\prime} \neq I$. Again by Proposition 6.3, it follows from (8.4) that there is $t^{\prime \prime} \in\left(\bar{\Omega}_{B} S\right)^{I}$ with $t^{\prime}=b t^{\prime \prime}$ and

$$
\mathrm{V} \models v_{1} b \cdot t^{\prime \prime}=u_{1} \quad \text { and } \quad \mathrm{V} \models v_{2}=t^{\prime \prime} \cdot a u_{2} .
$$

This implies the existence of a path in $\Gamma_{\psi}$ from $\omega\left(\delta_{j}\right)$ to $\alpha\left(\varepsilon_{j}\right)$, which once more leads to a contradiction with the definition of a transition edge.

Therefore, we have $t^{\prime}=I$, and so, thanks to Proposition 6.3, from (8.4) we get $\mathrm{V} \models v_{1}=u_{1}, a=b$ and $\mathrm{V} \models v_{2}=u_{2}$. This yields $\varepsilon_{j}=\delta_{j}$, which contradicts the initial assumption. Therefore, the set (8.1) is empty. In particular, $\varepsilon_{n}=\delta_{n}$ holds. Since $\varepsilon_{n}$ is the last transition edge for $u$, we have $\omega\left(\delta_{n}\right)=(\psi(u), I)$, which means that $\delta_{n}$ is the last transition edge for $v$, whence $m=n$ and $\varepsilon_{i}=\delta_{i}$ for every $i \in\{1, \ldots, n\}$. This concludes the proof that $\psi^{K(\ell l)}(u)=\psi^{K(\ell l)}(v)$.

Now, let $\zeta$ be an arbitrary continuous homomorphism from $\bar{\Omega}_{B} S$ into $S_{\psi}^{K(\ell \mathrm{l})}$. Because $\psi^{K(\ell \mathrm{l})}$ is onto, there is a continuous endomorphism $\lambda$ of $\bar{\Omega}_{B} \mathrm{~S}$ such that $\zeta=\psi^{K(\ell l)} \circ \lambda$. Since we also have $\mathrm{V} \models \lambda(u)=\lambda(v)$, we deduce that $\zeta(u)=\zeta(v)$. This establishes our claim that $S_{\psi}^{K(\ell \mathrm{l})} \models u=v$.

Applying Lemma 8.2, we conclude that $S_{\varphi}^{K(\ell l)} \models u=v$. By Reiterman's Theorem [17, we deduce that $S_{\varphi}^{K(\ell l)} \in \mathrm{V}$, thus proving that $\mathrm{V}$ is closed under the two-sided Karnofsky-Rhodes expansion.

Corollary 8.4. Let $\mathrm{V}$ be a pseudovariety of semigroups. The following conditions are equivalent:

(1) $\mathrm{V}$ is equidivisible and it is not contained in $\mathrm{CS}$;

(2) $\mathrm{V}=\mathrm{LI}$ (m) $\mathrm{V}$;

(3) $\mathrm{V}$ is closed under the two-sided Karnofsky-Rhodes expansion;

(4) $\mathrm{V}$ is closed under the two-sided connected expansion.

Proof. The equivalence (2) $\Leftrightarrow(3)$ is Corollary 3.3 . In particular, a pseudovariety closed under the the two-sided Karnofsky-Rhodes expansion contains LI. As $\mathrm{LI}$ is not contained in CS, the equivalences (10) $\Leftrightarrow(2) \Leftrightarrow(3)$ then follow from Theorem 8.3. Since the two-sided connected expansion is a quotient of the two-sided Karnofsky-Rhodes expansion, we clearly have (3) $\Rightarrow$ (44). Conversely, suppose that $\mathrm{V}$ is closed under the two-sided connected expansion. By Proposition 4.3, $\mathrm{V}$ is equidivisible. We claim that $\mathrm{V}$ is not contained in CS. Consider the mapping $\varphi$ from $\bar{\Omega}_{\{a\}} \mathrm{S}$ onto the trivial semigroup $S=\{1\}$. The path in $\Gamma_{\varphi}$ from $(I, 1)$ to $(1, I)$ labeled $a$ intersects precisely two strongly connected components of $\Gamma_{\varphi}$, while, for every $k \geq 2$, the path in $\Gamma_{\varphi}$ from $(I, 1)$ to $(1, I)$ labeled $a^{k}$ intersects precisely three strongly connected components of $\Gamma_{\varphi}$. Therefore, denoting by $\varphi^{C}$ the canonical continuous homomorphism from $\bar{\Omega}_{\{a\}} \mathrm{S}$ to $S^{C}$ whose restriction to $\{a\}^{+}$is the connected expansion of $\left.\varphi\right|_{\{a\}^{+}}$, we have $\varphi^{C}(a) \neq \varphi^{C}\left(a^{\omega+1}\right)$. This shows 
that $S^{C} \notin \mathrm{CR}$, establishing the claim, and concluding the proof that we have (4) $\Rightarrow(1)$.

There is one important further connection of the conditions of Corollary 8.4 with varieties of languages. Indeed, as has been proved by Pin 14 (cf. [15, Theorem 7.3]), the language counterpart of the operator $\mathrm{V} \mapsto \mathrm{LI}$ (m) V is the closure under unambiguous product.

We conclude the paper with one further application of our results for pseudovarieties of local groups. Combining Corollary 8.4 with Theorem 7.2 , we obtain the following.

Corollary 8.5. If $\mathrm{V}$ is a subpseudovariety of $\mathrm{LG}$ containing $\mathrm{LI}$ then $\mathrm{V}=$ LI $m$ V.

It is well known that $\mathrm{LI} \vee \mathrm{H}=\mathrm{LI}(\mathrm{m}) \mathrm{H}$ for every pseudovariety $\mathrm{H}$ of groups [11, Corollary 3.2]. The previous results provide an indirect proof of the following extension of that fact, which appears to be new.

Corollary 8.6. If $\mathrm{V}$ is a subpseudovariety of $\mathrm{LG}$ then $\mathrm{LI} \vee \mathrm{V}=\mathrm{LI} \times \mathrm{O} \mathrm{V}$.

Proof. Let $\mathrm{V}$ be a subpseudovariety of LG. Then the pseudovariety LI $\vee \mathrm{V}$ is equidivisible by Theorem 7.2. Therefore, applying Corollary 8.5, we obtain LI $\vee V=L I m(L I \vee V) \supseteq L I m \vee \supseteq L I \vee V$.

Reading [8, Corollary 4.3] one finds the following basis for LI $\vee$ CS:

$$
\text { LI } \vee C S=\llbracket z^{\omega}(x y)^{\omega} x t^{\omega}=z^{\omega} x t^{\omega}, x y^{\omega} z=\left(x y^{\omega} z\right)^{\omega+1} \rrbracket .
$$

As an example of application of Corollary [8.6, we obtain a simplified basis for LI $\vee C S$, made of a pseudoidentity involving only three letters.

Proposition 8.7. The pseudovariety LIVCS is defined by the pseudoidentity $(x y)^{\omega}(x z)^{\omega}(x y)^{\omega}=(x y)^{\omega}$.

Proof. By [5, Theorem 6.1], taking $\Sigma=\left\{(z t)^{\omega} z=z\right\}, \mathrm{H}=\mathrm{I}, W=\left\{x_{1}\right\}$, $m=0$, and $\alpha_{1}=x_{1}^{\omega+1}$, we obtain that the pseudovariety LI $m$ CS is defined by the following pseudoidentities:

$$
\begin{aligned}
\left(\left(x(z t)^{\omega} z y\right)^{\omega} x z y\left(x(z t)^{\omega} z y\right)^{\omega}\right)^{\omega} & =\left(x(z t)^{\omega} z y\right)^{\omega} \\
(x z y)^{\omega-1}\left(x(z t)^{\omega} z y\right)^{\omega+1}(x z y)^{\omega} & =(x z y)^{\omega} .
\end{aligned}
$$

Note that the first of these pseudoidentities is valid in LG while the pseudovariety defined by the second one is contained in LG. Hence, LI ${ }_{0}$ CS is defined by the pseudoidentity (8.7). Further simplifications of the pseudoidentity (8.7) may be carried out as follows. First, since it defines a subpseudovariety of $L G$, the $(\omega+1)$-power of the infinite element in the middle may be replaced by the base of that power. Second, pre-multiplying both sides by $z y$, postmultiplying by $x(z y x)^{\omega-1}$ and applying suitable conjugations to shift infinite powers, we obtain the pseudoidentity $(z y x)^{\omega}(z t)^{\omega}(z y x)^{\omega}=(z y x)^{\omega}$. Substituing $x$ by $z y$, we deduce the equivalent pseudoidentity $(z y)^{\omega}(z t)^{\omega}(z y)^{\omega}=$ $(z y)^{\omega}$, as the former can be recovered by substituting in the latter $y$ by $y x$. Renaming variables, this shows that $(x y)^{\omega}(x z)^{\omega}(x y)^{\omega}=(x y)^{\omega}$ is a simplified basis for LI 13 CS. Finally, we apply Corollary 8.6. 


\section{REFERENCES}

1. J. Almeida, Finite semigroups and universal algebra, World Scientific, Singapore, 1995, English translation.

2. __ Profinite semigroups and applications, Structural theory of automata, semigroups and universal algebra (New York) (V. B. Kudryavtsev and I. G. Rosenberg, eds.), Springer, 2005, pp. 1-45.

3. J. Almeida and A. Costa, Infinite-vertex free profinite semigroupoids and symbolic dynamics, J. Pure Appl. Algebra 213 (2009), 605-631.

4. J. Almeida and O. Klíma, Representations of relatively free profinite semigroups, irreducibility, and order primitivity, Tech. report, Univ. Masaryk and Porto, 2015, arXiv:1509.01389.

5. J. Almeida, S. Margolis, B. Steinberg, and M. Volkov, Characterization of group radicals with an application to mal'cev products, Illinois J. Math. 54 (2010), 199-221.

6. L. Chaubard, J.-E. Pin, and H. Straubing, Actions, wreath products of $\mathcal{C}$-varieties and concatenation product, Theor. Comp. Sci. 356 (2006), 73-89.

7. A. Costa, Semigrupos profinitos e dinâmica simbólica, Ph.D. thesis, Univ. Porto, 2007.

8. J. C. Costa, Some pseudovariety joins involving locally trivial semigroups, Semigroup Forum 64 (2002), 12-28.

9. S. Eilenberg, Automata, languages and machines, vol. B, Academic Press, New York, 1976.

10. G. Z. Elston, Semigroup expansions using the derived category, kernel, and Malcev products, J. Pure Appl. Algebra 136 (1999), no. 3, 231-265.

11. T. E. Hall and P. Weil, On radical congruence systems, Semigroup Forum 59 (1999), 56-73.

12. F. W. Levi, On semigroups, Bull. Calcutta Math. Soc. 36 (1944), 141-146.

13. J. D. McKnight, Jr. and A. J. Storey, Equidivisible semigroups, J. Algebra 12 (1969), $24-48$.

14. J.-E. Pin, Propriétés syntactiques du produit non ambigu, Automata, languages and programming (Proc. Seventh Internat. Colloq., Noordwijkerhout, 1980) (Berlin), Lect. Notes in Comput. Sci., vol. 85, Springer, 1980, pp. 483-499.

15. _ Syntactic semigroups, Handbook of Formal Languages (G. Rozenberg and A. Salomaa, eds.), Springer, 1997.

16. J.-E. Pin, H. Straubing, and D. Thérien, Locally trivial categories and unambiguous concatenation, J. Pure Appl. Algebra 52 (1988), 297-311.

17. J. Reiterman, The Birkhoff theorem for finite algebras, Algebra Universalis 14 (1982), $1-10$.

18. J. Rhodes and B. Steinberg, Profinite semigroups, varieties, expansions and the structure of relatively free profinite semigroups, Int. J. Algebra Comput. 11 (2002), 627-672.

19. Springer, 2009.

20. J. Rhodes and B. Tilson, The kernel of monoid morphisms, J. Pure Appl. Algebra 62 (1989), 227-268.

21. J. Rhodes and P. Weil, Decomposition techniques for finite semigroups, using categories II, J. Pure Appl. Algebra 62 (1989), 285-312.

22. I. Simon, Hierarchies of events of dot-depth one, Ph.D. thesis, Univ. Waterloo, 1972.

23. H. Straubing, Aperiodic homomorphisms and the concatenation product of recognizable sets, J. Pure Appl. Algebra 15 (1979), 319-327.

CMup, Departamento de Matemática, Faculdade de Ciências, Universidade do Porto, Rua do Campo Alegre 687, 4169-007 Porto, Portugal.

E-mail address: jalmeida@fc.up.pt

CMUC, Department of Mathematics, University of Coimbra, Apartado 3008, EC Santa Cruz, 3001-501 Coimbra, Portugal.

E-mail address: amgc@mat.uc.pt 